\title{
Conjunctival intraepithelial microcyst
}

Ch'ng Tun Wang', Ong Poh Yan²

${ }^{1}$ Department of Ophthalmology, Hospital Raja Permaisuri Bainun, Ipoh, Perak, Malaysia; ${ }^{2}$ Department of Ophthalmology, Hospital Selayang, Selangor, Malaysia

\section{Clinical context}

A 17-year-old teenage girl with open-angle glaucoma as a complication of penetrating keratoplasty had undergone an uneventful primary augmented trabeculectomy surgery. One year postsurgery, her intraocular pressure (IOP) was stable in the low teens with a shallow diffuse bleb.

\section{Question 1}

Describe the findings in Figure $1 \mathrm{a}$ and $1 \mathrm{~b}$.

\section{Question 2}

What is the implication of the presence of the findings in Figure $1 \mathrm{a}$ and $1 \mathrm{~b}$ in trabeculectomy surgery? 


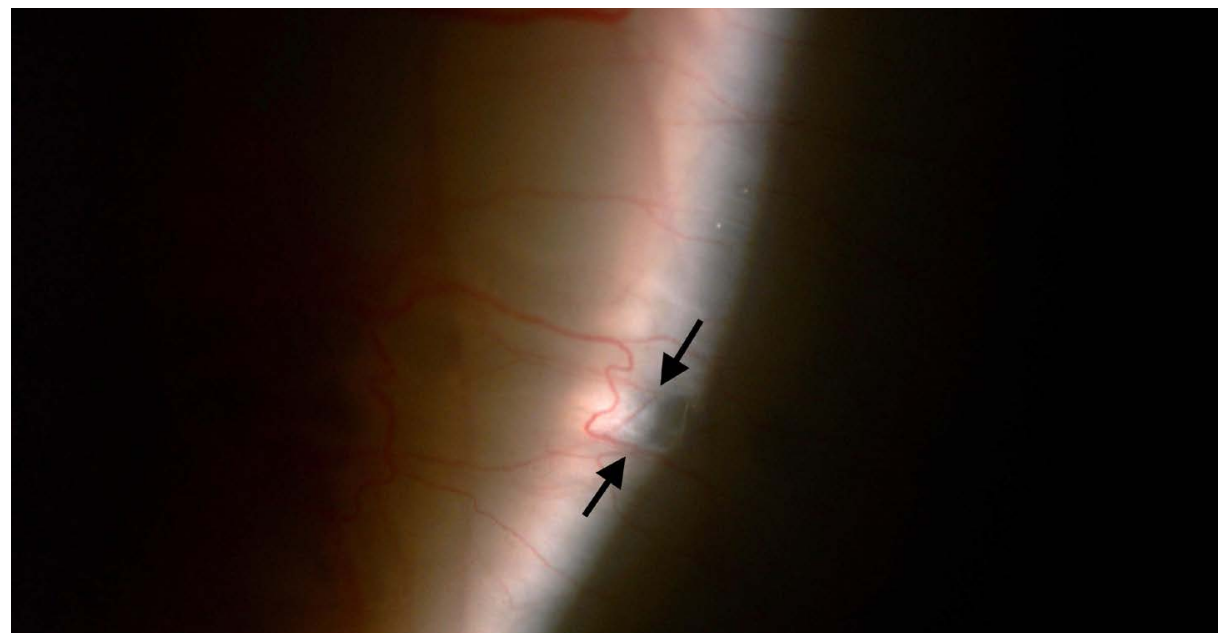

Fig. 1a.

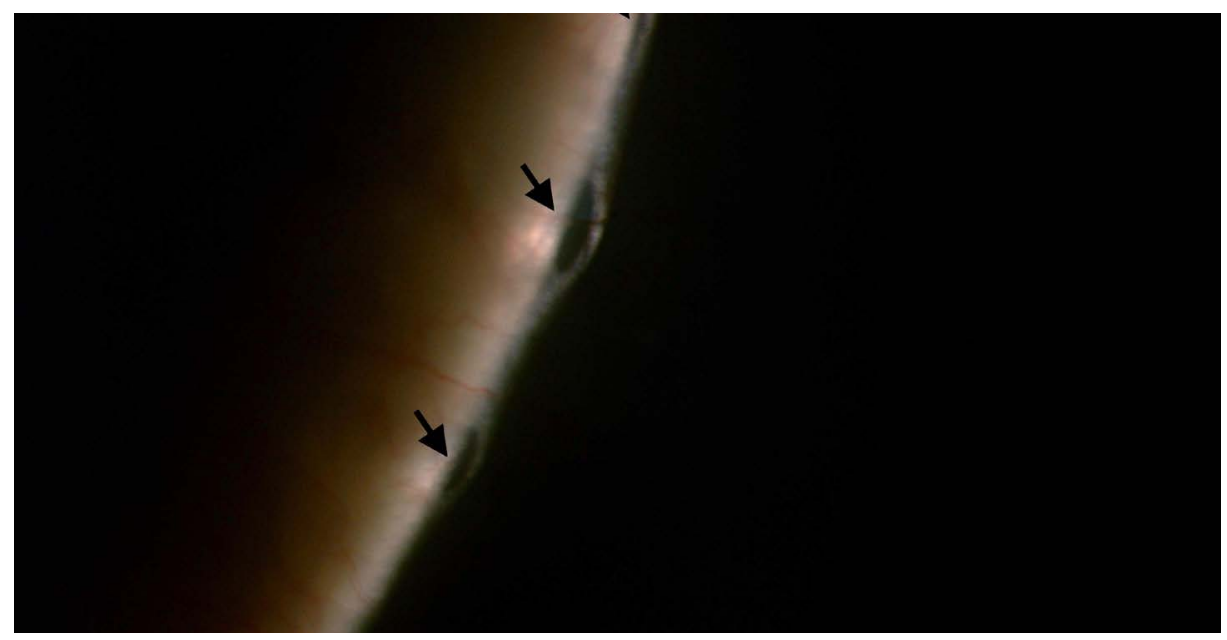

Fig. $1 b$. 


\section{Answer 1}

Slit lamp examination revealed multiple conjunctival intraepithelial microcysts. As seen in Figure 1a, the parallelepiped illumination of the slit lamp shows a conjunctival intraepithelial microcyst. In Figure 1b, specular reflection technique of the microcysts shows the cross-section of tiny cystic vesicles present on the outer surface of the conjunctiva. The microcysts consist of various sizes of cystic cavities covered by a thin layer of conjunctiva with conjunctival vessels overlying it.

\section{Answer 2}

Conjunctival intraepithelial microcysts are associated with lower IOP and are an indicator of successful glaucoma surgery. ${ }^{1-2}$ It is possible these microcysts act as mini-reservoirs for aqueous to leave the conjunctival vessels via the transconjunctival route.

\section{References}

1. Arjunan Kumaran, Rahat Husain, Hla M. Htoon, Tin Aung. Longitudinal Changes in Bleb Height, Vascularity, and Conjunctival Microcysts After Trabeculectomy. J Glaucoma 2018;27:578-584

2. Meziani L, Tahiri Joutei Hassani R, El Sanhar- awi M, et al. Evaluation of blebs after filtering surgery with en-face anterior-segment optical coherence tomography: a pilot study. J Glaucoma. 2016;25:550-558. 